\title{
The Topography of Tactile Working Memory
}

\author{
Justin A. Harris, Irina M. Harris, and Mathew E. Diamond \\ Cognitive Neuroscience Sector, International School for Advanced Studies, Trieste, Italy 34014
}

To investigate the contribution of topographically organized brain areas to tactile working memory, we asked human subjects to compare the frequency of two vibrations presented to the same fingertip or to different fingertips. The vibrations ranged from 14 to $24 \mathrm{~Hz}$ and were separated by a retention interval of variable length. For intervals $<1 \mathrm{sec}$, subjects were accurate when both vibrations were delivered to the same fingertip but were less accurate when the two vibrations were delivered to different fingertips. For 1 or 2 sec intervals, subjects performed equally well when comparing vibrations delivered either to the same finger or to corresponding fingers on opposite hands, but they performed poorly when the vibrations were delivered to distant fingers on either hand. These results suggest that working memory resides within a topographic framework. As a further test, we performed an experiment in which the two comparison vibrations were presented to the same fingertip but an interference vibration was presented during the retention interval. The interpolated vibration disrupted accuracy most when delivered to the same finger as the comparison vibrations and had progressively less effect when delivered to more distant fingers. We conclude that topographically organized regions of somatosensory cortex contribute to tactile working memory, possibly by holding the memory trace across the retention interval. One stimulus can be accurately compared with the memory of a previous stimulus if they engage overlapping representations, but activation of the common cortical territory by an interpolated stimulus can disrupt the memory trace.

Key words: somatosensory; cortex; flutter vibration; SI; SII; human; finger
This study is part of an inquiry into how sensory cortical regions contribute to perceptual learning and memory (Diamond et al., 2001; Harris et al., 2001b). Important evidence has come from demonstrations that perceptual learning can be spatially restricted in a way that corresponds to the topographic organization of sensory cortical processing areas. For example, human subjects that had learned to recognize a visual stimulus presented at a specific retinotopic location failed to recognize the stimulus at other locations (Karni and Sagi, 1991; Fahle, 1994; Ahissar and Hochstein, 1996, 1997; Schoups and Orban, 1996; Dill and Fahle, 1997, 1998). This topography strongly implicates primary visual cortex in which neurons have the requisite retinotopic selectivity to account for the spatial localization of performance.

Recently, we examined whether perceptual learning for tactile information is also topographic (Harris et al., 2001a). Using only one fingertip, subjects learned to discriminate between two stimuli, i.e., either two vibrations of different frequency, two punctate stimuli of different force, or two surfaces of different roughness. After completion of training, we tested the subjects with other fingertips and identified a clear topography of the learned discriminative ability. Indeed, the learned information was spatially localized in a way that was best accounted for by the topographic organization of somatosensory cortical areas, and in particular, SI and SII. Moreover, these experiments tested the following additional prediction of the topographic learning hypothesis: because sensory cortex is parcelled into multiple areas, each specialized

\footnotetext{
Received May 31, 2001; revised July 10, 2001; accepted July 25, 2001.

This work was supported by a fellowship from the Italian Ministry of Universities and Scientific and Technological Research to J.A.H. and grants from the Telethon Foundation, Consiglio Nazionale delle Ricerche, and the James S. McDonell Foundation.

Correspondence should be addressed to Dr. Justin Harris, Cognitive Neuroscience Sector, International School for Advanced Studies, Via Beirut, 2-4, 34014 Trieste, Italy. E-mail: jharris@sissa.it.

Copyright (C) 2001 Society for Neuroscience $0270-6474 / 01 / 218262-08 \$ 15.00 / 0$
}

for processing specific types of stimuli (Kaas, 1993), the spatial distribution of learning for a given type of tactile stimulus should reflect the specific somatotopic organization of the relevant cortical area. Consistent with this prediction, we found different topographic distributions for learning the vibration discrimination versus learning the pressure and roughness discriminations (Harris et al., 2001a); these differences correspond to the peripheral and cortical segregation of vibration processing from pressure and roughness processing (Johnson and Hsiao, 1992). We concluded that, in each task, the information was stored in stimulus-specific cortical fields, each characterized by a different receptive field organization, feature selectivity, and callosal connectivity.

The present experiments investigated whether short-term or "working" memory for tactile information resides within a topographic framework, just as the long-term changes that underlie recognition seem to. Working memory refers to the ability to hold information across short time spans (usually on the order of seconds) for subsequent manipulation or comparison with new information (Baddeley, 1996). Most investigations into the neural bases of working memory have emphasized the contribution of prefrontal cortical areas in the executive processes of selecting and monitoring the information held in memory (Ungerleider et al., 1998; Levy and Goldman-Rakic, 2000; Petrides, 2000a; Fuster, 2001). However, some investigators have suggested that posterior sensory and association regions of cortex may also contribute to working memory, particularly in the short-term storage or maintenance of mnemonic information (Zhou and Fuster, 1996; Courtney et al., 1997; Postle and D’Esposito, 1999; Postle et al., 1999, 2000; Petrides, 2000b; Druzgal and D'Esposito, 2001). The present study was designed to test the hypothesis that somatosensory cortex is an essential part of the circuit supporting working memory for tactile information. We reasoned that the hypothesis 

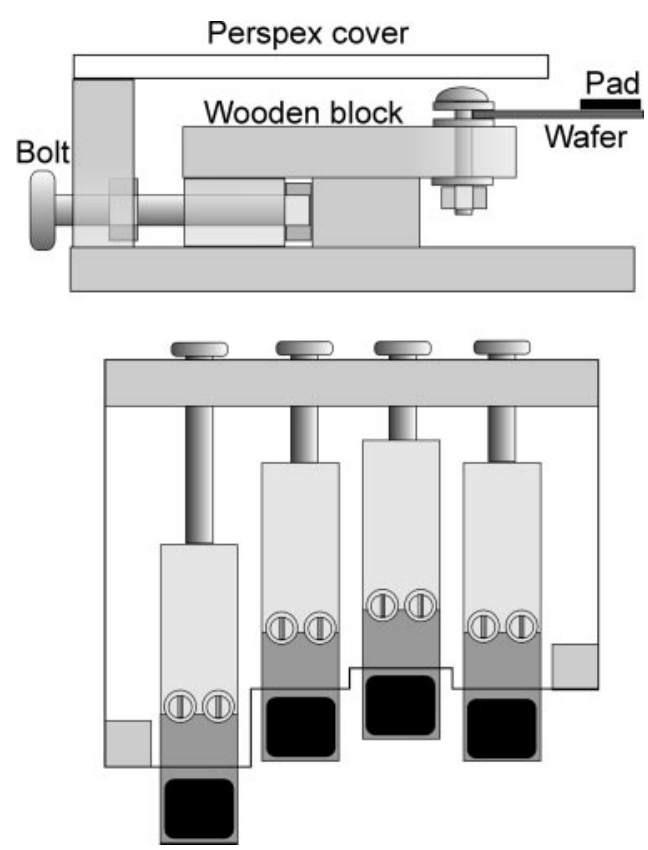

Figure 1. Diagram of the apparatus, as viewed from the side (top) and from above (bottom). Piezoelectric wafers were secured with plastic bolts to wooden blocks. Each block and wafer was held in place by a bolt passing through the back of the apparatus. The bolts kept the blocks in alignment but allowed adjustment of the position of the wafers along the front-back axis. The apparatus was covered by a piece of Perspex.

would be supported if performance on a tactile working memory task were to show the same topography as somatosensory cortex itself.

\section{MATERIALS AND METHODS}

\section{Subjects}

A total of 31 subjects, 12 females and 19 males, participated in the study. They ranged in age from 24 to 45 years, and four were left-handed. Five of the subjects participated in two experiments. All subjects were financially compensated for their time (12,000 Italian lire per hour). Recruitment of subjects and experimental procedures were conducted in accordance with the Declaration of Helsinki.

\section{Materials}

The vibrations were produced using piezoelectric wafers (length, $38 \mathrm{~mm}$; width, $19 \mathrm{~mm}$; thickness, $0.5 \mathrm{~mm}$; Morgan Matroc, Bedford, OH) individually driven by $80 \mathrm{~V}$ pulses from custom-built amplifiers controlled by a computer running Labview (National Instruments, Austin, TX). A flat rubber pad (length, $19 \mathrm{~mm}$; width, $19 \mathrm{~mm}$; thickness, $2 \mathrm{~mm}$ ) was glued on the top face at the outer end of each wafer. Two identical (but mirrorimage) cases were constructed to house four wafers each (Fig. 1). Inside these, the wafers were individually mounted on wooden blocks, aligned side-by-side, and spaced $25 \mathrm{~mm}$ apart (center-to-center), with the ends of the wafers protruding from the housing case. The position of each wafer could be adjusted to accommodate the lengths of each subject's fingers. Two padded wooden blocks (length, $60 \mathrm{~mm}$; width, $100 \mathrm{~mm}$; height, 45 $\mathrm{mm}$ ) were used as wrist supports.

\section{Experimental designs and objectives}

In each of four experiments, the subjects compared two serially presented vibrations, in the range of $14-24 \mathrm{~Hz}$, and verbally reported whether they were of the same or different frequencies. The pair of vibrations to be evaluated varied across trials, forcing subjects to compare the two vibrations rather than make a categorical judgment about one of them (cf. Hernández et al., 1997). To determine the most suitable parameters in this task, experiment 1 examined subjects' performances across three different interstimulus intervals (ISIs) $(1,2$, and $5 \mathrm{sec}$ ) and at three different levels of difficulty, corresponding to frequency discrim-
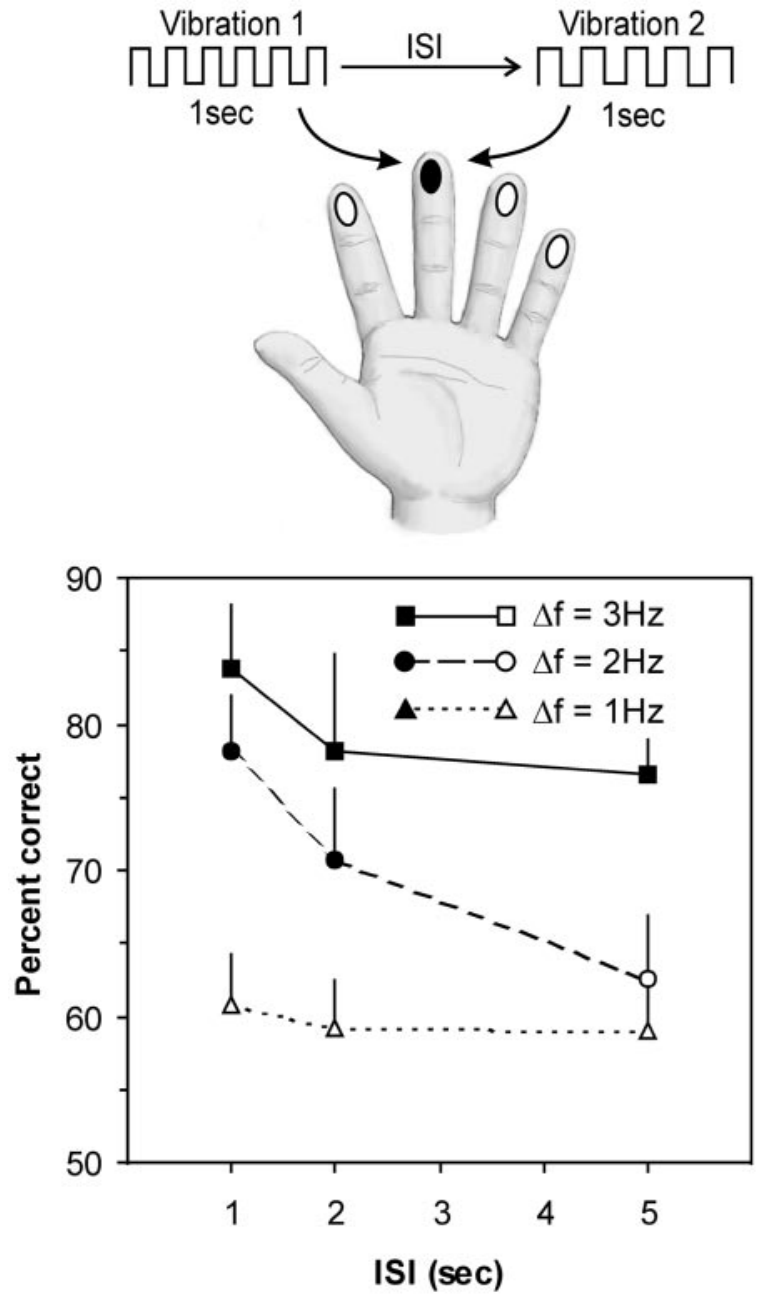

Figure 2. Design and results of experiment 1. On each trial, subjects felt two $1 \mathrm{sec}$ long vibrations in the range $14-24 \mathrm{~Hz}$, separated by an interstimulus interval (ISI) of 1,2 , or $5 \mathrm{sec}$. The vibrations were delivered to any of the eight fingers (excluding thumbs), but both vibrations were delivered to the same finger within a given trial (the example illustrated here is for the middle finger on the left hand). On half of the trials, the vibrations were the same frequency; on the remaining trials, their frequency differed $(\Delta f)$ by 2,4 , or $6 \mathrm{~Hz}$. Their mean accuracy (percentage correct) for each ISI and $\Delta f$ is shown in the graph. Filled symbols represent scores significantly above chance ( $50 \%$ correct) by $z$ test at $p<0.05$. Error bars represent SEM.

inations of 2, 4, or $6 \mathrm{~Hz}$. On the basis of the results obtained (Fig. 2), all subsequent experiments used a frequency difference of $4 \mathrm{~Hz}$ and ISIs no $>2$ sec. Experiment 2 had two objectives: first, to extend the findings of experiment 1 by examining tactile working memory across very short retention intervals (ISIs between $200 \mathrm{msec}$ and $1 \mathrm{sec}$ ); and second, to assess the interhemispheric distribution of tactile working memory across these intervals by testing subjects with two vibrations presented either to the same finger or to corresponding fingers on opposite hands. Experiment 3 was a more detailed investigation of the topography of tactile working memory, examining how the performance varied when the two vibrations were delivered to the same finger or to fingers increasingly further apart. To determine whether the topography changes across the retention interval, ISIs of either 1 or $2 \mathrm{sec}$ were used. Finally, experiment 4 examined topography at a short retention interval (400 msec). In addition, this experiment investigated whether tactile working memory is subject to interference by an interpolated vibratory stimulus and whether this interference effect is also topographic. Thus, on half the trials, an interference vibration was presented during the retention interval at varying topographic distances from the two comparison vibrations. 


\section{Procedure}

Subjects were tested in a single session that lasted $50 \mathrm{~min}$ for experiments 1 and 2 and $60 \mathrm{~min}$ for experiments 3 and 4 . The subject was seated at a table in front of the two wafer cases and was instructed to rest the ball of each hand on the wooden blocks and place the pad of each finger (excluding the thumbs) on the rubber pads at the end of each wafer.

The frequency, measured as the number of deflections per second, was always an even number in the range of 14-24 Hz. Although it has been reported that changes in vibration frequency can influence the perception of stimulus intensity (LaMotte and Mountcastle, 1975), it appears that the complement is not generally true; we observed (our unpublished data) that subjects' accuracy at comparing frequency was not affected by shifts in vibration amplitude that caused the two vibrations to have equivalent intensity (i.e., by increasing the amplitude of a lower frequency). Therefore, in the present experiments the vibrations consisted of a square wave of fixed amplitude $(250 \mu \mathrm{m})$ and rise time $(5 \mathrm{msec})$. This amplitude is well above detection threshold (LaMotte and Mountcastle, 1975; Mountcastle et al., 1990), and thus ensured that each subject could reliably detect the vibrations at all fingertips.

Experiment 1. Six subjects participated in this experiment. On each trial they received two consecutive vibrations lasting $1 \mathrm{sec}$ each and had to judge whether the vibrations were of the same or different frequencies. The two vibrations were always delivered to the same fingertip, but the selected finger changed between trials (Fig. 2). The interval between trials was 2 sec.

The experiment used a $3 \times 3$ factorial design consisting of three levels of discrimination difficulty [determined by the difference in frequency $(\Delta f)$ between the two vibrations, either 2,4 , or $6 \mathrm{~Hz}$ ] and three different ISIs $(1,2$, or $5 \mathrm{sec})$. There were 32 trials in each of the nine experimental conditions, making a total of 288 trials. On half of the trials, the vibrations were of the same frequency; on the other half, they were different frequencies. Trials at the three ISIs were randomly intermixed. In contrast, trials at each of the three levels of difficulty were separated into different blocks (with each block containing an equal number of trials at each ISI). We did this because we expected that subjects would have difficulty adopting an appropriate decision rule if trials with different $\Delta f$ s were intermixed (e.g., vibrations differing by $2 \mathrm{~Hz}$ might be judged as being the same if they were intermixed among trials for which the vibrations differed by $6 \mathrm{~Hz}$ ). However, rather than have the subjects complete all trials from one level of difficulty before proceeding to the next block, we further split the trials into two blocks per level and intermixed these blocks. Thus, there were a total of six blocks, each comprising 64 trials. The order of the blocks was counterbalanced using an ABCCBA design with the exact order chosen randomly for each subject. Breaks of 5-10 min separated consecutive blocks.

Experiment 2. This experiment examined how accuracy at comparing vibrations varied across short ISIs $(200,400,600,800 \mathrm{msec}$, and $1 \mathrm{sec})$. The experiment also sought to determine whether accuracy differed depending on whether the vibrations were delivered to the same finger or to corresponding fingers on opposite hands (Fig. 3). Thus, the experiment used a 5 (ISI) $\times 2$ (finger) factorial design and proceeded in a manner similar to that of experiment 1 . On each trial, the subjects $(n=$ 10) felt two $1 \mathrm{sec}$ vibrations, with $\Delta f$ equal to either 0 or $4 \mathrm{~Hz}$, and they had to judge whether the vibrations were the same or different. There were 32 trials for each of the 10 conditions, randomly intermixed.

Experiment 3. The purpose of this experiment was to determine with more precision how subjects' accuracy in comparing two vibrations varied as a function of the topographic distance between the sites at which the vibrations were delivered. On each trial, the subjects $(n=10)$ felt two $1 \mathrm{sec}$ vibrations, with $\Delta f$ equal to either 0 or $4 \mathrm{~Hz}$, and had to judge whether the vibrations were the same or different. The experiment used a 3 (topographic distance between vibration sites) $\times 2$ (same hand vs different hands $) \times 2(1 \mathrm{sec}$ vs $2 \mathrm{sec}$ ISI $)$ factorial design. The topographic distance between the vibration sites (Fig. 4) was either "+0" (the vibrations were delivered to the same finger or to corresponding fingers on each hand), "+1" (the vibrations were delivered to neighboring fingers or to neighbors of corresponding fingers), or " +2 " (the vibrations were delivered to second neighbors or to second neighbors of corresponding fingers). The specific fingers involved changed across trials in a counterbalanced fashion. There were 32 trials in each of the 12 conditions, and trials from all conditions were randomly intermixed.

Experiment 4. The purpose of this experiment was twofold. First, to extend the findings of experiment 3 , this experiment investigated how
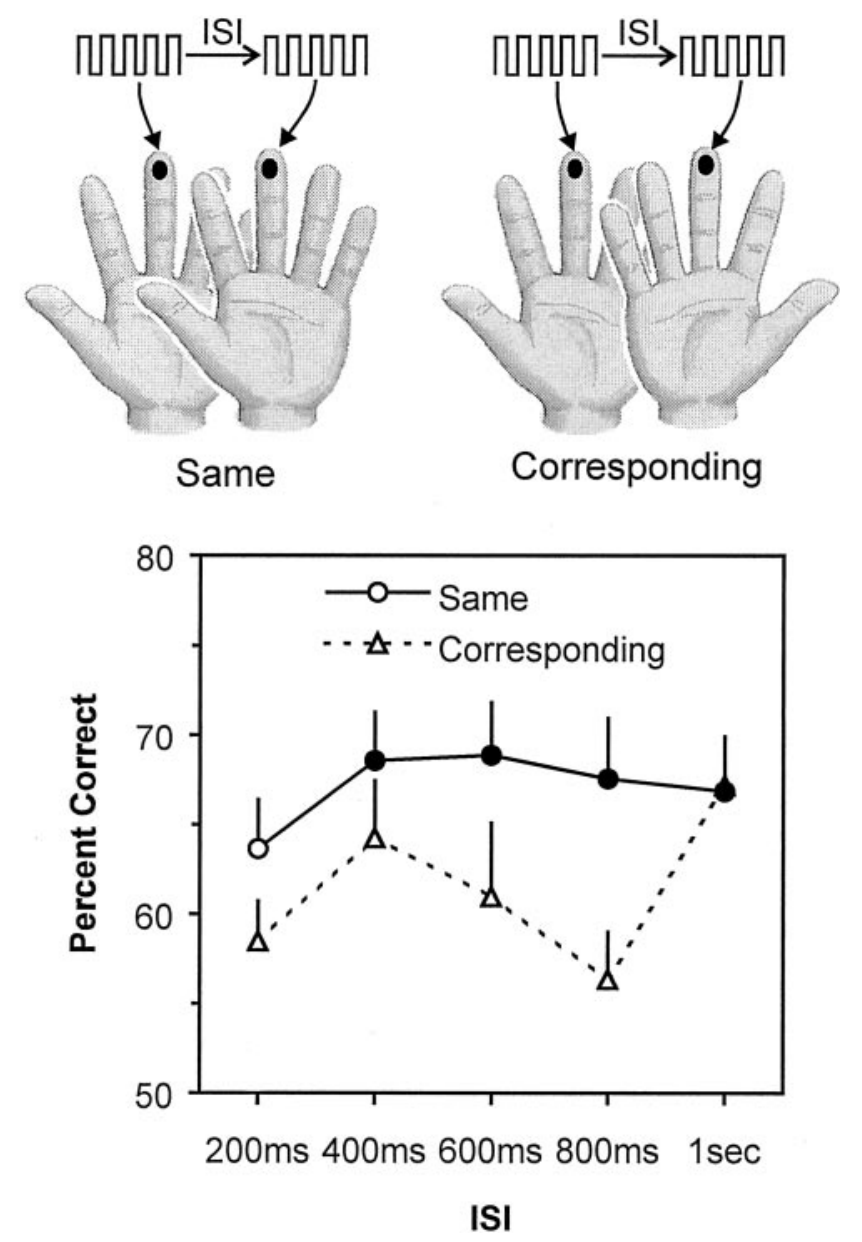

Figure 3. Design and results of experiment 2. On each trial, subjects felt two 1-sec-long vibrations separated by an interval (ISI) of 200, 400, 600, $800 \mathrm{msec}$, or $1 \mathrm{sec}$. The first vibration was delivered to any of the eight fingers excluding thumbs (e.g., the middle finger on the left hand in the illustration), and the second vibration was delivered either to the same finger as the first vibration or to the corresponding finger on the other hand. Their mean accuracy (percentage correct) for same-finger versus corresponding-finger comparisons at each ISI is shown in the graph. Filled symbols represent scores significantly above chance (50\% correct) by $z$ test at $p<0.05$. Error bars represent SEM.

topographic distance between stimulus sites affects accuracy across a very short retention interval (ISI, $400 \mathrm{msec}$ ). Second, to determine whether the working memory task could be disrupted by an interpolated tactile stimulus, on half of the trials an "interference" vibration was presented during the interval between the two comparison vibrations. More specifically, the experiment was designed to assess whether the interference effect might itself be topographically related to the location of the interpolated vibration relative to the comparison vibrations. The rationale was as follows: if the memory trace used to compare two vibrations were held in somatosensory cortex, then the impact of an interpolated stimulus should likewise be subject to the same topographic principle that regulates comparison of the target vibrations.

The experiment consisted of 12 conditions, each comprising 32 trials. On each trial, the subjects $(n=10)$ received three vibrations presented serially; two lasted for $1 \mathrm{sec}$, and one for $250 \mathrm{msec}$. The subjects were instructed to compare only the two long vibrations. All subjects reported that they had no difficulty in identifying which vibrations they had to compare. The long vibrations varied in the range $14-24 \mathrm{~Hz}$, whereas the $250 \mathrm{msec}$ vibration was always $24 \mathrm{~Hz}$. The ISI was $400 \mathrm{msec}$ for both intervals (i.e., between the first and second vibrations and between the second and third vibrations). Figure 5 summarizes the temporal sequence of the trials.

To examine tactile interference, on half of the trials the short vibration was interpolated between the two longer target vibrations (thus, the 

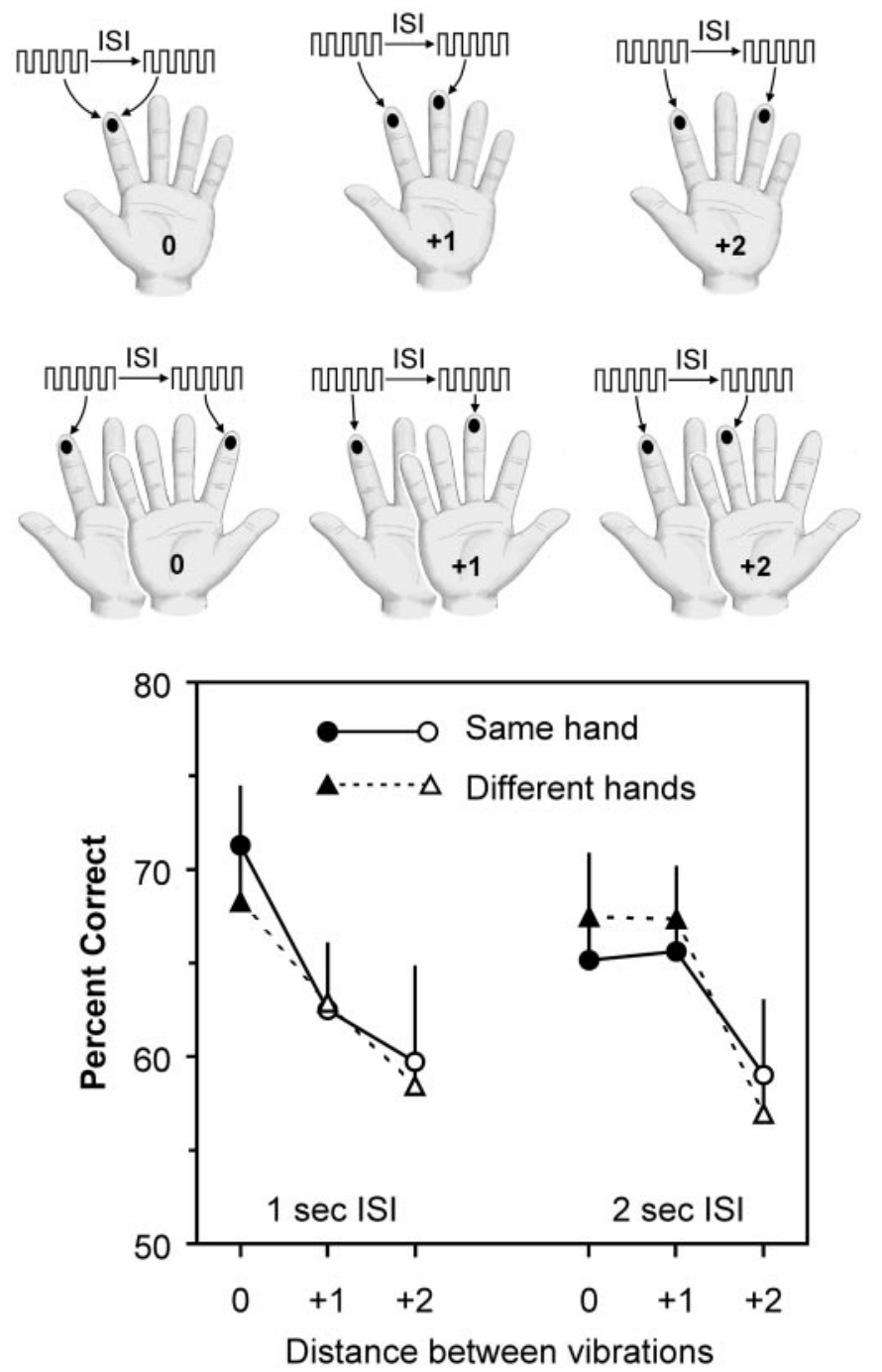

Figure 4. Design and results of experiment 3. On each trial, subjects felt two vibrations separated by an interval (ISI) of either 1 or $2 \mathrm{sec}$. The first vibration was delivered to any of the eight fingers excluding thumbs (e.g., the left index finger in the illustration). The second vibration was delivered to the same finger $(+0)$, its neighboring finger $(+1)$, its second neighbor $(+2)$ on the same hand (top row of diagrams), or to the corresponding finger $(+0)$, its neighbor $(+1)$, or its second neighbor $(+2)$ on the other hand (bottom row of diagrams). Their mean accuracy (percentage correct) for comparisons at different topographic distances for both ISIs is shown in the graph. Filled symbols represent scores significantly above chance (50\% correct) by $z$ test at $p<0.05$. Error bars represent SEM.

interval separating the two target vibrations was exactly $1050 \mathrm{msec}$ ). On these trials, the two target vibrations were always delivered to the same fingertip (which varied between trials), but the interference vibration could occur on the same finger $(+0)$ as the target vibrations, the neighboring finger $(+1)$, the second neighbor $(+2)$, or any of the three corresponding fingers on the opposite hand (see Fig. 5 for an example).

To examine the topography of tactile working memory at the short $(400 \mathrm{msec})$ retention interval, on half of the trials the short vibration occurred after both of the target vibrations. On these trials, the two target vibrations were delivered to the same finger $(+0)$, neighboring fingers $(+1)$, second neighbors $(+2)$, or any of these three combinations on different hands. The short vibration always occurred on the same finger as the first vibration. We assumed that, on these trials, the short vibration would not disrupt comparison of the target vibrations because of its presentation after the two target vibrations; the short vibration was included so that all trials in the experiment would have an equal number of vibrations and thus a single set of instructions would apply to all trials. These trials served as a replication of the design used in experiment 3 ,
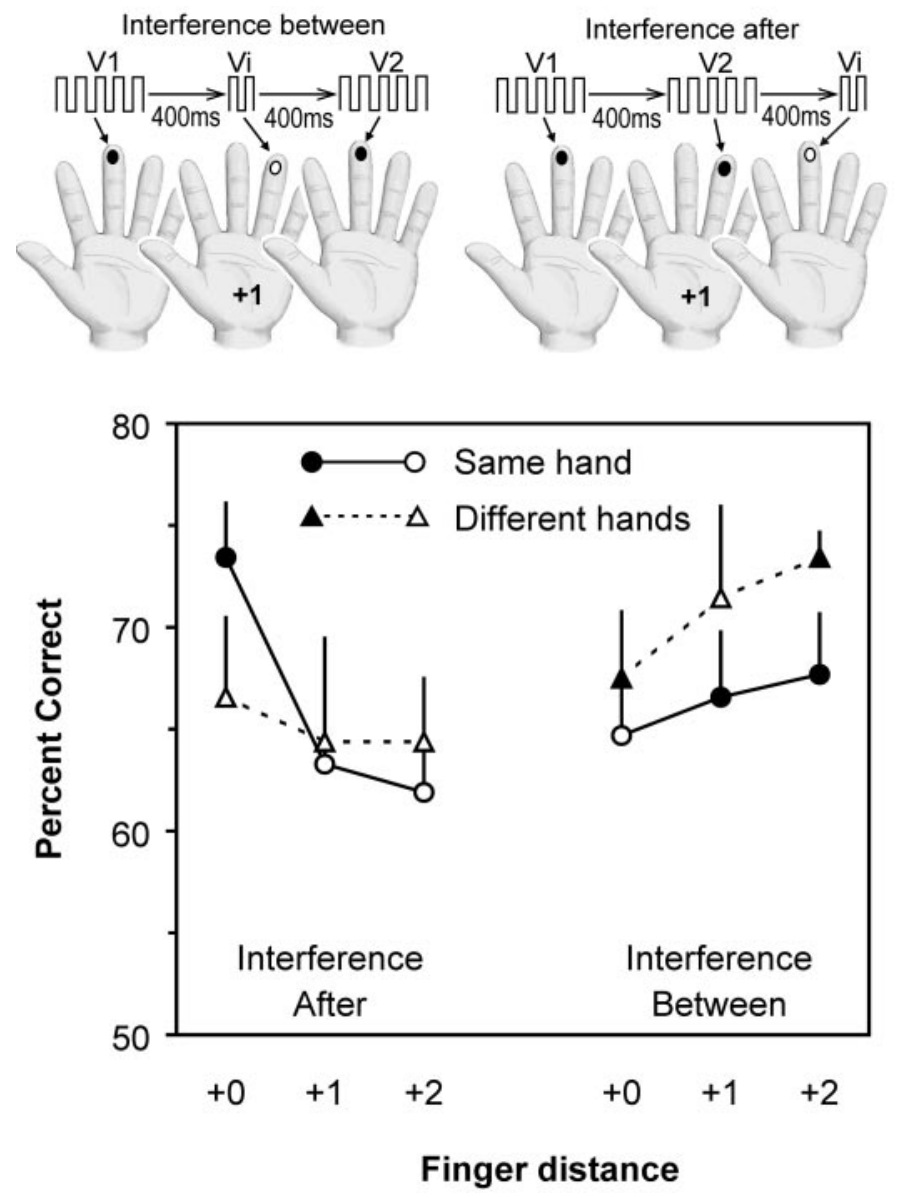

Figure 5. Design and results of experiment 4. On each trial, subjects felt three vibrations, each separated by $400 \mathrm{msec}$. Two of the vibrations (V1 and $V 2$ ) were $1 \mathrm{sec}$ long and served as the comparison stimuli; an additional vibration $(\mathrm{Vi})$ was $250 \mathrm{msec}$ long and served as an interference stimulus. On half of the trials (Interference After), $V i$ was presented after $V 1$ and $V 2$; on the remaining trials (Interference Between), $V i$ was presented between $V 1$ and $V 2$. The first vibration $(V 1)$ was delivered to any of the eight fingers excluding thumbs (e.g., the left middle finger in the illustration). The second vibration $(V 2$ or $V i)$ was delivered to the same finger $(+0)$, its neighboring finger $(+1$, as illustrated in this figure), its second neighbor $(+2)$ on the same hand, or to any of the corresponding fingers $(+0,+1,+2)$ on the other hand. The third vibration $(V i$ or $V 2)$ was delivered to the same fingertip as V1. Thus, on the interference-after trials, the two comparison vibrations were separated by varying topographic distances, whereas on the interference-between trials, the comparison vibrations were always on the same finger $(+0$ distance), but the interpolated $V i$ was separated from these by varying topographic distances. Their mean accuracy (percentage correct) for each condition is shown in the graph. Filled symbols represent scores significantly above chance ( $50 \%$ correct) by $z$ test at $p<0.05$. Error bars represent SEM.

except that the ISI between comparison vibrations was $400 \mathrm{msec}$. These trials also served a second important purpose: because they were randomly intermixed with trials in which the interference stimulus occurred between the target vibrations, on a given trial, the subjects could not know in advance whether the second vibration would be the interference vibration or the second comparison vibration. This ensured that the subjects attended to the interference stimulus when it occurred between the comparison vibrations.

Statistical analyses. For each experiment, a repeated measures ANOVA was conducted to identify significant effects for each factor. In addition, the accuracy scores for each condition were subjected to a $z$ test to determine whether they were significantly above chance (50\% correct). For both analyses, $\alpha$ was set at 0.05 . 


\section{RESULTS}

\section{Experiment 1}

This experiment examined accuracy across three different ISIs (1, 2 , and $5 \mathrm{sec}$ ) and at three different levels of difficulty, corresponding to $\Delta f$ of 2,4 , or $6 \mathrm{~Hz}$. Subjects were more accurate for larger $\Delta f$ s, and their accuracy tended to decrease at the longer retention intervals (Fig. 2). Specifically, when $\Delta f$ was $2 \mathrm{~Hz}$, the subjects were barely above chance at deciding whether the vibrations were the same or different, and their performance did not change as a function of retention interval ( $z<1.33$; $p>0.09$, at all ISIs). Overall, the subjects were more accurate when $\Delta f$ was $4 \mathrm{~Hz}$, but their accuracy declined as the retention interval increased and was particularly poor at the longest retention interval (at $1 \mathrm{sec}$ ISI, $z=3.18, p<0.001$; at $2 \mathrm{sec}$ ISI, $z=1.88, p=0.03$; at $5 \mathrm{sec}$ ISI, $z=1.27, p>0.1$ ). Finally, the subjects were uniformly accurate when $\Delta f$ was $6 \mathrm{~Hz}$, even as the ISI increased $(z>1.84 ; p<0.05$, at all ISIs). The ANOVA confirmed that there was a significant main effect for frequency difference $\left(F_{(2,10)}=25.02 ; p<0.001\right)$, but there was not a main effect for retention interval $\left(F_{(2,10)}=\right.$ $2.72 ; p=0.114)$ nor was there a significant interaction between these factors $\left(F_{(2,10)}=1.92 ; p=0.146\right)$.

From these results, we concluded that a $2 \mathrm{~Hz}$ difference could not be reliably identified. Conversely, a $6 \mathrm{~Hz}$ difference appears to have been too easy, because the subjects' accuracy was close to ceiling. In contrast, the subjects could identify the $4 \mathrm{~Hz}$ difference, yet their performance was not at ceiling. Furthermore, when the vibrations differed by $6 \mathrm{~Hz}$, the subjects continued to perform accurately across the longest retention interval $(5 \mathrm{sec})$, whereas their performance dropped substantially at this interval when the vibrations differed by only $4 \mathrm{~Hz}$. This distinction implies that the subjects may have used different strategies to compare the vibrations in the two conditions. For example, when the difference was large $(6 \mathrm{~Hz})$, an effective method to compare the vibrations may have involved classifying each one with a verbal label (e.g., "low" or "high" frequency). Because such labels can be held in working memory for a long period (Baddeley, 1996), accuracy would remain high across long retention intervals. In contrast, the "labeling" strategy would have been less effective for finer discriminations (i.e., when the difference was $4 \mathrm{~Hz}$ ), forcing subjects to rely on a sensory memory trace of the first stimulus; under these conditions, accuracy would be limited by the relatively short decay time of such nonverbal memory traces ( $\mathrm{Lu}$ et al., 1992; Baddeley, 1996). On the basis of these results, we decided to use the $4 \mathrm{~Hz}$ difference as the frequency comparison for all subsequent experiments.

\section{Experiment 2}

This experiment had two goals: first, to examine tactile working memory across very short retention intervals (ISIs between 200 msec and $1 \mathrm{sec}$ ); and second, to assess the interhemispheric distribution of tactile working memory across these intervals by testing subjects with two vibrations presented either to the same finger or to corresponding fingers on opposite hands. At all ISIs $<1 \mathrm{sec}$, the subjects were more accurate at comparing vibrations delivered to the same finger than vibrations delivered to corresponding fingers on different hands (Fig. 3). When the ISI was 1 sec, the subjects were equally accurate with the same finger versus corresponding fingers on different hands.

$Z$ tests confirmed that, when the vibrations were delivered to the same finger, accuracy was not significantly above chance for judgments made with at an ISI of $200 \operatorname{msec}(z=1.60 ; p=0.055)$, but were above chance at all other ISIs (in all cases, $z>1.67 ; p<$
0.05). When the vibrations were delivered to corresponding fingers on opposite hands, accuracy was significantly above chance if the ISI was $1 \sec (z=2.16 ; p=0.015)$ but not at any of the shorter ISIs (in all cases, $z<1.53$; $p>0.06$ ). In addition, the ANOVA revealed a significant main effect for stimulus site (same finger versus corresponding opposite finger; $F_{(1,9)}=19.35 ; p=0.002$ ), but no main effect for ISI $\left(F_{(4,36)}=2.41 ; p=0.067\right)$ and no significant interaction between these factors $\left(F_{(4,36)}=1.88 ; p=\right.$ $0.136)$. Nonetheless, it is clear from the Figure that the difference between same and corresponding fingers is confined to ISIs $<1$ sec; at $1 \mathrm{sec}$, accuracy was identical for the two conditions.

\section{Experiment 3}

Here, we tested more stimulus sites to explore the topography of working memory in finer detail. At the $1 \mathrm{sec}$ ISI, performance on the task was strongly topographic inasmuch as the subjects' accuracy decreased as the distance between the vibration sites increased (Fig. 4). Specifically, the subjects were most accurate when the two vibrations were delivered to the same finger or to corresponding fingers on different hands (finger distance $=+0$ ), and scores on both of these conditions were above chance $(z=$ 2.23 and $2.41 ; p=0.013$ and 0.008 , respectively). The subjects were less accurate when the vibrations were delivered to neighboring fingers on the same hand or to neighbors of corresponding fingers on different hands $(+1)$, and were least accurate when the vibrations were delivered to second neighbors on the same hand or on different hands $(+2)$. None of these conditions produced scores above chance (in all cases, $z<1.2$; $p>0.1$ ).

When the ISI was $2 \mathrm{sec}$, accuracy was strikingly similar for vibrations delivered to the same hand or to different hands, as was found for the $1 \mathrm{sec}$ ISI. However, unlike the case for the shorter ISI, subjects were equally accurate for vibrations delivered to the same or corresponding fingers $(+0)$ or to the first neighbors of these fingers $(+1)$; scores for all four conditions were above chance (in all cases, $z>1.80 ; p<0.05$ ). Accuracy was lowest for vibrations delivered to second neighbors $(+2)$ on either same or different hands $(z=0.79$ and $0.57 ; p>0.2)$, as was found for the $1 \mathrm{sec}$ ISI.

The topographic pattern across fingers was confirmed by the ANOVA, which showed that there was a significant main effect of finger distance $\left(F_{(2,18)}=11.28 ; p=0.001\right)$. There was no main effect of same versus different hands $\left(F_{(1,9)}<1\right)$, nor was there interaction between hand and finger distance $\left(F_{(1,9)}<1\right)$, confirming that the subjects' performance in comparing vibrations between hands was equivalent to their performance when comparing within one hand. Furthermore, the analysis showed that the performances at 1 and $2 \mathrm{sec}$ ISIs were not different overall $\left(F_{(1,9)}<1\right)$ and performance with the same versus different hands remained similar across both ISIs $\left(F_{(1,9)}<1\right)$. The analysis did not find a significant interaction between ISI and finger distance $\left(F_{(2,18)}=2.48 ; p=0.112\right)$ when all three finger positions were taken into account $(+0,+1$, and +2$)$. We decided to carry out a post hoc test to determine the significance of the apparent "broadening" of topography from the +0 distance to the +1 distance (bilaterally) as ISI increased. Any such change might have been concealed in the test for interaction between ISI and finger distance (above) by the poor performance at the +2 distance, which was common to both ISIs. The post hoc analysis identified a significant interaction between ISI and finger distance $\left(F_{(1,9)}=\right.$ $5.65 ; p=0.041)$, provided that only positions +0 and +1 were evaluated. In sum, we conclude that accuracy in comparing vibrations was affected by finger distance and performance across 
the two hands was equivalent. Furthermore, as ISI increased from 1 to $2 \mathrm{sec}$, topography changed such that subjects became equally accurate for vibrations delivered to the +0 and +1 positions (on either hand).

\section{Experiment 4}

Here, we examined how working memory across a short retention interval $(400 \mathrm{msec})$ varied as a function of the topographic distance between the comparison vibrations, and we concurrently probed the topography of the working memory trace by presenting an interference stimulus in the interval between the two comparison vibrations. On trials in which the short vibration occurred after the two target vibrations (Fig. 5, left), the subjects' accuracy showed a topographic pattern comparable in some respects to that observed in experiment 3 (compare Figs. 4, 5). For example, subjects were more accurate when comparing vibrations on the same finger $(+0)$ than when comparing vibrations delivered to neighboring fingers $(+1)$ or to second neighbors $(+2)$ on the same hand. However, the bilateral symmetry observed in experiment 3 was less apparent. In particular, subjects were less accurate for vibrations delivered to corresponding opposite fingers than for vibrations on the same finger. The apparent discrepancy is easily explained by recalling that the short ISI in the present experiment resembles that of experiment 2, in which subjects were less accurate for comparisons between opposite fingers at ISIs $<1 \mathrm{sec}$. The $z$ tests showed that the scores were significantly above chance only when the vibrations were on the same finger $(z=2.85 ; p=0.002)$, and not for any other combination of fingers (in all cases, $z \leq 1.5 ; p>0.065$ ).

The effect of the interference vibration interpolated between the two target vibrations also revealed a topographic pattern (Fig. 5 , right). Recall that the two comparison vibrations were delivered to the same finger. When the interference stimulus occurred on the same finger as the target vibrations, the subjects were least accurate, and their scores were not significantly above chance $(z=1.54 ; p=0.062)$. In contrast, the subjects' scores were above chance when the interference stimulus was delivered to a different finger $(z \geq 1.68 ; p<0.05$, except when the interference stimulus was at the +1 distance on the different hand, for which $z=1.57 ; p=0.058$, because of the large variance in this condition). Importantly, the effect of the interference was a uniform function of finger distance; the further the interference vibration site was from the comparison vibration sites, the less it interfered with the subjects' performance.

An ANOVA showed that there were no significant main effects for when the interference vibration occurred (after vs between, $F_{(1,9)}<1$ ), hand (same vs different, $F_{(1,9)}=1.56 ; p=0.24$ ), or finger distance $\left(F_{(2,18)}<1\right)$. There was, however, a significant interaction between interference (after vs between) and finger distance $\left(F_{(2,18)}=8.69 ; p=0.002\right)$. This interaction confirms that accuracy decreased with increasing distance between the comparison stimuli (on the "interference-after" trials), whereas accuracy increased with increasing distance separating the interference vibration site from the comparison vibrations (on the "interference-between" trials). The interaction between interference and hand was not significant $\left(F_{(1,9)}=2.78 ; p=0.13\right)$, nor was the interaction between hand and finger distance $\left(F_{(2,18)}<1\right)$.

To directly ascertain the net effect of the interference vibration at each finger location, we used as a reference the subjects' scores on trials in which the relative position of the comparison vibration was +0 on the same hand and the interference occurred after the comparison stimuli (average accuracy $=73.4 \%$ ) (Fig. 5). We compared this with trials in which the interference was delivered between the two comparison stimuli (recall that the comparison stimuli were always delivered to the same finger on the interference-between trials). Each comparison was tested using a one-tailed Student's $t$ test. As is evident in Figure 5, there was no detectable interference effect of the interpolated vibration when delivered at +1 or +2 finger distances on the opposite hand $(p=$ 0.32 and 0.5 , respectively). In contrast, an effect was evident when the interpolated vibration was delivered at any of the other finger distances. However, the statistical analysis only found a significant effect when the interpolated vibration was delivered to the same finger as the comparison vibrations $(p=0.015)$. The effect fell short of statistical significance when the interpolated vibration was delivered at +1 or +2 distances on the same hand or to +0 finger on the other hand $(p=0.061,0.065$, and 0.080 , respectively).

In sum, by studying the effect of an interpolated interference vibration, this experiment joins the preceding ones in showing that working memory for tactile stimuli, under the testing conditions used here, resides within a topographic framework.

\section{DISCUSSION}

We examined people's ability to compare the frequency of two vibrations that were delivered serially to the fingertips. The vibrations were separated by a temporal interval, forcing the subjects to hold a memory of the first vibration across the interval to compare it with the second vibration. Below, we will argue that the resulting data are most consistent with the idea that a critical component of the network that holds the memory trace across the retention interval resides in the topographically organized somatosensory cortical areas.

The principal result is that accuracy at comparing two vibrations was determined by the somatotopic distance between the locations of the vibrations; when the two vibrations were delivered to the same finger, accuracy was high, and when they were delivered to different fingers, the subjects' accuracy decreased as the distance between the vibration sites increased. This topography implies a role for somatosensory areas of cortex because these have tactile receptive fields that conserve information about stimulus site (Merzenich et al., 1978; Kaas and Pons, 1988; Iwamura et al., 1993; Gelnar et al., 1998; Maldjian et al., 1999; Francis et al., 2000). Moreover, these areas contain large numbers of neurons with cutaneous receptive fields that encode low-frequency vibrotactile stimulation (Mountcastle et al., 1969, 1990; Merzenich et al., 1978; Kaas and Pons, 1988; Burton and Sinclair, 1991; Iwamura et al., 1993; Francis et al., 2000; Hernández et al., 2000; Salinas et al., 2000). Indeed, the association between SI neuronal activity and vibrotactile sensation is sufficiently strong that electrical stimulation of neurons in SI at a particular frequency produces sensations that monkeys treat as identical to a mechanical vibration of that frequency delivered to the fingertip (Romo et al., 1998, 2000). Thus, we suggest that neurons in SI and/or SII not only encode the vibrations but also contribute to the process by which the memory of one vibration frequency is compared with the perceived frequency of a subsequent vibration.

In experiment 1 , subjects performed very poorly when $\Delta f$ was 2 $\mathrm{Hz}$, whereas they performed at near ceiling level when $\Delta f$ was 6 $\mathrm{Hz}$; this led us to set $\Delta f$ at $4 \mathrm{~Hz}$ for all subsequent experiments. Having selected this parameter, we found that subjects could perform the comparison for delay intervals up to $2 \mathrm{sec}$ (provided 
that both stimuli were presented to the same fingertip), but not for an interval of $5 \mathrm{sec}$. This result suggests that the tactile memory trace underlying a difficult comparison has a decay time very similar to that of other perceptual or sensory memories and it contrasts with the more enduring working memory traces of verbal information (Lu et al., 1992; Baddeley, 1996).

The precise topographic pattern for the working memory task varied depending on how long the memory trace had to be maintained. The most notable difference was between comparisons made across short intervals $(<1 \mathrm{sec})$ versus longer intervals ( 1 or $2 \mathrm{sec}$ ). When the interval was short, the subjects were better at comparing stimuli delivered to the same finger than stimuli delivered to any combination of different fingers. When the interval was longer, the subjects were as accurate for vibrations delivered to corresponding fingers on each hand as for vibrations delivered to the same finger. In other words, the capacity to compare stimuli was initially confined to one finger but became bilateral when the interval was $1 \mathrm{sec}$ or more. Another change in topography appeared between the 1 and 2 sec intervals; at $1 \mathrm{sec}$, accuracy was higher for same-finger or corresponding opposite finger comparisons than for comparisons between neighboring fingers, but this difference was no longer apparent at the $2 \mathrm{sec}$ interval.

The change from unilateral to bilateral topography as the interval increased, together with the broadening of the topography to the +1 position, suggests that different regions of somatosensory cortex might be involved in retaining the tactile working memory trace at different times. For example, the single-finger topography observed at the short $(<1 \mathrm{sec})$ intervals suggests that an essential component of the tactile memory trace was held in a cortical field in which neurons have small (single-digit) receptive fields and are not connected to the hand representation of the opposite hemisphere. These criteria point to SI, and in particular to Brodmann areas $3 b$ and 1 . In the hand representation in monkeys, most area $3 \mathrm{~b}$ and 1 neurons have single-digit receptive fields (Merzenich et al., 1978; Iwamura et al., 1993), and there are virtually no interhemispheric projections through the corpus callosum (Killackey et al., 1983). The somatotopic organization of SI in humans has been established by functional imaging experiments showing that this area contains detailed representations of the contralateral hand (Gelnar et al., 1998; Maldjian et al., 1999; Francis et al., 2000). Thus, one might expect that neurons in SI could only support the comparison of two vibrations delivered to the same finger, which would account for the data we collected for short ISIs.

The bilaterally symmetric topography observed at the 1 and 2 sec intervals suggests that an essential component of the tactile memory trace was held in a somatotopically organized cortical field with strong interhemispheric connections. These criteria point to SII (Brodmann's area 43), which contains neurons that accurately encode vibration frequencies in the range used here (Salinas et al., 2000). Importantly, there are strong callosal projections connecting homotopic regions of SII in each hemisphere (Killackey et al., 1983; Manzoni et al., 1984), and neurons in SII have receptive fields that typically include more than one finger and are often bilaterally symmetric (Jiang et al., 1997; Gelnar et al., 1998; Maldjian et al., 1999; Francis et al., 2000; Disbrow et al., 2001). Thus, these neurons should be able to support the comparison of vibrations delivered to corresponding fingers on different hands or even neighboring fingers on the same hand.

The conclusion that some combination of somatosensory cortical regions contributes to comparing vibrations is supported by a recent neurophysiological study in which monkeys performed a working memory task similar to that used here (Salinas et al., 2000). The study identified neurons in SII for which activity encoded the frequency of the first vibration and maintained that activity during the retention interval between the two vibrations. It is tempting to speculate that the maintained activity constituted the physiological correlate of the working memory trace. However, these same researchers found no evidence of maintained neuronal activity in SI across the course of the retention interval [although other researchers have reported such evidence in monkeys holding tactile working memories for roughness (Zhou and Fuster, 1996)]. The discrepancy between the sharp topography at short retention intervals that is suggestive of SI involvement (the present report) and the absence of maintained neuronal activity in SI (Salinas et al., 2000) may be attributable to differences in the retention intervals used in these two studies. Specifically, the monkeys in the Salinas et al. (2000) study were always required to compare vibrations across intervals $>1 \mathrm{sec}$, whereas in the present report we have observed that the sharp topography indicating an SI storage site dissipates as the retention interval approaches $1 \mathrm{sec}$. In other words, SI neurons may play a crucial role in retention of tactile working memories only across short intervals. Alternatively, as suggested by Salinas et al. (2000), working memory for vibrations might be supported by activity in SII, and not SI, irrespective of the retention interval. In this case, our observation of a shift from unilateral to bilateral representation of the memory trace as the retention interval reached $1 \mathrm{sec}$ may reflect the transfer of the relevant information between the SII regions in each hemisphere. Although $1 \mathrm{sec}$ would seem excessively long for such transfer, this may be the time required to transfer and build up a precise representation of the temporal characteristics of the vibration among neurons in the ipsilateral SII.

What role might neurons in somatosensory cortex play in working memory for vibrations? One possibility is that these neurons contribute to holding the memory trace of the first vibration across the retention interval so that it can be compared with the second vibration. We tested this by reasoning that, if the comparison relies on a sensory memory trace for the first vibration that is held by neurons in somatosensory cortex, then presentation of an interference vibration that activates those same cortical neurons should disrupt the memory trace and so prevent accurate comparison. The impact of the interference vibration should decrease when presented at somatotopic locations further away from the two comparison vibrations. Support for this contention comes from experiment 4 in which a brief vibration interpolated between the comparison vibrations did interfere with the subjects' accuracy; moreover, the interference was most severe when delivered to the same fingertip as the two comparison vibrations, and its influence decreased systematically at more distant fingertips. Thus, the interference effect seems to depend on the interpolated vibration activating the same territory in somatosensory cortex that had been activated by the first of the two comparison vibrations.

By concluding that neurons in somatosensory cortex contribute to maintaining the working memory trace for a vibrotactile stimulus, we do not wish to argue that other cortical areas do not also contribute to this process. Many studies have provided strong evidence implicating regions of prefrontal cortex in working memory (for review, see Levy and Goldman-Rakic, 2000; Petrides, 2000a; Fuster, 2001). In particular, a study of working memory for vibrotactile stimuli in monkeys identified populations 
of prefrontal neurons in which firing rate encoded the frequency of the first of two vibrations and that maintained this firing pattern across intervals of several seconds (Romo et al., 1999). We propose that prefrontal neurons act in conjunction with anterior parietal neurons to sustain a tactile working memory trace. Several areas in prefrontal cortex are directly connected with SII (Morecraft et al., 1992; Carmichael and Price, 1995; Cavada et al., 2000). Thus, transient excitatory assemblies could be established to bridge prefrontal cortex and SII, serving to maintain activity in the somatosensory neurons in the absence of external sensory input (cf. Sarnthein et al., 1998; Fuster, 2001).

Although many crucial details remain to be elucidated, all findings presented here point toward a model for the neural basis of tactile working memory whereby the network that retains sensory information during a delay period is not segregated into special "memory centers," but instead is distributed across the very same sensory cortical regions responsible for "on-line" representation of the stimuli.

\section{REFERENCES}

Ahissar M, Hochstein S (1996) Learning pop-out detection: specificities to stimulus characteristics. Vision Res 36:3487-3500.

Ahissar M, Hochstein S (1997) Task difficulty and the specificity of perceptual learning. Nature 387:401-406.

Baddeley A (1996) The fractionation of working memory. Proc Natl Acad Sci USA 93:13468-13472.

Burton H, Sinclair RJ (1991) Second somatosensory cortical area in macaque monkeys: neural responses to punctate vibrotactile stimulation of the glabrous skin on the hand. Brain Res 538:127-135.

Carmichael ST, Price JL (1995) Sensory and premotor connections of the orbital and medial prefrontal cortex of macaque monkeys. J Comp Neurol 363:642-664.

Cavada C, Compañy T, Tejedor J, Cruz-Rizzolo RJ, Reinoso-Suárez F (2000) The anatomical connections of the macaque monkey orbitofrontal cortex: a review. Cereb Cortex 10:220-242.

Courtney SM, Ungerleider LG, Keil K, Haxby JV (1997) Transient and sustained activity in a distributed neural system for human working memory. Nature 386:608-611.

Diamond ME, Petersen RS, Harris JA (2001) Sensory learning and the brain's body map. In: The somatosensory system: deciphering the brain's body image (Nelson RJ, ed). Boca Raton, FL: CRC, in press.

Dill M, Fahle M (1997) The role of visual field position in patterndiscrimination learning. Proc R Soc Lond B Biol Sci264:1031-1036.

Dill M, Fahle M (1998) Limited translation invariance of human visual pattern recognition. Percept Psychophys 60:65-81.

Disbrow E, Roberts T, Poeppel D, Krubitzer L (2001) Evidence for interhemispheric processing of inputs from the hands in human S2 and PV. J Neurophysiol 85:2236-2244.

Druzgal TJ, D'Esposito M (2001) Activity in fusiform face area modulated as a function of working memory load. Brain Res Cogn Brain Res $10: 355-364$.

Fahle M (1994) Human pattern recognition: parallel processing and perceptual learning. Perception 23:411-427.

Francis ST, Kelly EF, Bowtell R, Dunseath WJ, Folger SE, McGlone F (2000) fMRI of the responses to vibratory stimulation of the digit tips. NeuroImage 11:188-202.

Fuster JM (2001) The prefrontal cortex-an update: time is of the essence. Neuron 30:319-333.

Gelnar PA, Krauss BR, Szeverenyi NM, Apkarian AV (1998) Fingertip representation in the human somatosensory cortex: an fMRI study. NeuroImage 7:261-283.

Harris JA, Harris IM, Diamond ME (2001a) The topography of tactile learning in humans. J Neurosci 21:1056-1061.

Harris JA, Petersen RS, Diamond ME (2001b) The cortical distribution of sensory memories. Neuron 30:315-318.

Hernández A, Salinas E, García R, Romo R (1997) Discrimination in the sense of flutter: new psychophysical measurements in monkeys. J Neurosci 17:6391-6400.

Hernández A, Zainos A, Romo R (2000) Neuronal correlates of sensory discrimination in the somatosensory cortex. Proc Natl Acad Sci USA 97:6191-6196.

Iwamura Y, Tanaka M, Sakamoto M, Hikosaka O (1993) Rostrocaudal gradients in the neuronal receptive field complexity in the finger region of the alert monkey's postcentral gyrus. Exp Brain Res 92:360-368.

Jiang W, Tremblay F, Chapman E (1997) Neuronal encoding of texture changes in the primary and the secondary somatosensory cortical areas of monkeys during passive texture discrimination. J Neurophysiol $77: 1656-1662$.

Johnson KO, Hsiao SS (1992) Neural mechanisms of tactual form and texture perception. Annu Rev Neurosci 15:227-250.

Kaas JH (1993) Evolution of multiple areas and modules within neocortex. Perspect Dev Neurobiol 1:101-107.

Kaas JH, Pons TP (1988) The somatosensory system of primates. In: Comparative primate biology, Vol 4, Neurosciences (Steklis H, ed), pp 421-468. New York: Alan Liss.

Karni A, Sagi D (1991) Where practice makes perfect in texture discrimination: evidence for primary visual cortex plasticity. Proc Natl Acad Sci USA 88:4966-4970.

Killackey HP, Gould 3rd HJ, Cusick CG, Pons TP, Kaas JH (1983) The relation of corpus callosum connections to architectonic fields and body surface maps in sensorimotor cortex of new and old world monkeys. J Comp Neurol 219:384-419.

LaMotte RH, Mountcastle VB (1975) Capacities of humans and monkeys to discriminate between vibratory stimuli of different frequency and amplitude: a correlation between neural events and psychophysical measurements. J Neurophysiol 38:539-559.

Levy R, Goldman-Rakic PS (2000) Segregation of working memory functions within the dorsolateral prefrontal cortex. Exp Brain Res 133:23-32.

Lu ZL, Williamson SJ, Kaufman L (1992) Behavioral lifetime of human auditory sensory memory predicted by physiological measures. Science $258: 1668-1670$

Maldjian JA, Gottschalk A, Patel RS, Detre JA, Alsop DC (1999) The sensory somatotopic map of the human hand demonstrated at $4 \mathrm{~T}$. NeuroImage 10:55-62.

Manzoni T, Barbaresi P, Conti F (1984) Callosal mechanism for the interhemispheric transfer of hand somatosensory information in the monkey. Behav Brain Res 11:155-170.

Merzenich MM, Kaas JH, Sur M, Lin CS (1978) Double representation of the body surface within cytoarchitectonic areas $3 \mathrm{~b}$ and 1 in "SI" in the owl monkey (Aotus trivirgatus). J Comp Neurol 181:41-73.

Morecraft RJ, Geula C, Mesulam MM (1992) Cytoarchitecture and neural afferents of orbitofrontal cortex in the brain of the monkey. J Comp Neurol 323:341-358.

Mountcastle VB, Talbot WH, Sakata H, Hyvarinen J (1969) Cortical neuronal mechanisms in flutter-vibration studied in unanesthetized monkeys. Neuronal periodicity and frequency discrimination. J Neurophysiol 32:452-484.

Mountcastle VB, Steinmetz MA, Romo R (1990) Frequency discrimination in the sense of flutter: psychophysical measurements correlated with post-central events in the behaving monkey. J Neurosci 10:3032-3044.

Petrides M (2000a) The role of the mid-dorsolateral prefrontal cortex in working memory. Exp Brain Res 133:44-54.

Petrides M (2000b) Dissociable roles of mid-dorsolateral prefrontal and anterior inferotemporal cortex in visual working memory. J Neurosci 20:7496-7503.

Postle BR, D'Esposito MD (1999) "What"-then-"where" in visual working memory: an event-related fMRI study. J Cognit Neurosci 11:585-597.

Postle BR, Berger JS, D'Esposito MD (1999) Functional neuroanatomical double dissociation of mnemonic and executive control processes contributing to working memory performance. Proc Natl Acad Sci USA 96:12959-12964.

Postle BR, Stern CE, Rosen BR, Corkin S (2000) An fMRI investigation of cortical contributions to spatial and nonspatial visual working memory. NeuroImage 11:409-423.

Romo R, Hernandez A, Zainos A, Salinas E (1998) Somatosensory discrimination based on cortical microstimulation. Nature 392:387-390.

Romo R, Brody CD, Hernandez A, Lemus L (1999) Neuronal correlates of parametric working memory in the prefrontal cortex. Nature 399:470-473.

Romo R, Hernandez A, Zainos A, Brody CD, Lemus L (2000) Sensing and touching: psychophysical performance based on cortical microstimulation. Neuron 26:273-278.

Salinas E, Hernández A, Zainos A, Romo R (2000) Periodicity and firing rate as candidate neural codes for the frequency of vibrotactile stimuli. J Neurosci 20:5503-5515.

Sarnthein J, Petsche H, Rappelsberger P, Shaw GL, von Stein A (1998) Synchronization between prefrontal and posterior association cortex during human working memory. Proc Natl Acad Sci USA 95:7029-7096.

Schoups AA, Orban GA (1996) Interocular transfer in perceptual learning of a pop-out discrimination task. Proc Natl Acad Sci USA 93:7358-7362.

Ungerleider LG, Courtney AM, Haxby JV (1998) A neural system for human visual working memory. Proc Natl Acad Sci USA 95:883-890.

Zhou YD, Fuster JM (1996) Mnemonic neuronal activity in somatosensory cortex. Proc Natl Acad Sci USA 93:10533-10537. 